HD-THEP-03-30

\title{
Resummed effective action in the world-line formalism
}

\author{
Thomas Auer*, Michael G. Schmidt ${ }^{\dagger}$ AND Claus Zahlten ${ }^{\ddagger}$ \\ Institut für Theoretische Physik \\ Universität Heidelberg \\ Philosophenweg 16, D-69120 Heidelberg, Germany
}

\begin{abstract}
Using the world-line method we resum the scalar one-loop effective action. This is based on an exact expression for the one-loop action obtained for a background potential and a Taylor expansion of the potential up to quadratic order in $\mathrm{x}$-space. We thus reproduce results of Masso and Rota very economically. An alternative resummation scheme is suggested using "center of mass" based loops which is equivalent under the assumption of vanishing third and higher derivatives in the Taylor expansion but leads to simplified expressions. In an appendix some general issues concerning the relation between world-line integrals with fixed end points versus integrals with fixed center are clarified. We finally note that this method is also very valuable for gauge field effective actions where it is based on the Euler-Heisenberg type resummation.
\end{abstract}

\footnotetext{
*e-mail: thomas.t.auer@web.de

†e-mail: m.g.schmidt@thphys.uni-heidelberg.de

‡e-mail: zahlten@thphys.uni-heidelberg.de
} 


\section{Introduction}

In this paper we reconsider the one-loop contribution to the effective action for a scalar theory with self-interaction potential $V(\phi(x))$. Based on the momentum space method of ref. [1] E. Masso and F. Rota have shown that the terms up to quadratic order in the Taylor expansion of the potential can be resummed exactly [2].

In the framework of the world-line formalism [3, 4, 5] this fact is not surprising: world-line path integrals can be computed analytically when the action contains only terms of at most quadratic order in the world-line fields. In addition to that, in general the world-line formalism has some valuable advantages in calculating effective actions: the outer fields can be taken into account as a background in representation space easily which leads to very compact and elegant expressions.

Both of these facts suggest that the exact resummation of quadratic terms performed in ref. 2] should be possible in a much more straight forward way using world-line methods.

The paper is organized as follows. In section 2 we calculate the resummed one-loop contribution to the effective action using world-line methods. We confirm the result obtained in [2] and show that indeed within the world-line formalism the calculation demands much less effort. In section 3 we suggest an alternative resummation scheme using the freedom to choose a different organization of the paths to be integrated over inherent to the world-line formalism. This results in a very efficient way of resumming terms up to second order and leads to simplified expressions. Section 4 is devoted to the relation between the two resummation schemes and to a short comparison of both results with the invers mass expansion of the effective action given in refs. [6, 7]. Finally, in section 5 we show how the presented formalism can equally well be applied to the case of gauge fields and conclude our results. In the appendix we discuss some general issues concerning the relation between world-line path integrals with fixed end points versus integrals with fixed "center of mass".

\section{Resummation of the effective action}

In Euclidean space time conventions the world-line representation of the one-loop contribution to the effective action reads (see e.g. [5] )

$$
\Gamma^{(1)}[\varphi]=\frac{1}{2} \int_{0}^{\infty} \frac{d T}{T} e^{-m^{2} T} \int d^{D} q \int_{x(0)=q}^{x(T)=q} \mathcal{D} x \exp \left\{-\int_{0}^{T} d \tau\left[\frac{1}{4} \dot{x}^{2}(\tau)+V^{\prime \prime}(\varphi(x))\right]\right\}
$$

We will now Taylor expand the self-interaction potential, absorb the terms up to the quadratic order into the free path integral and resum the one-loop contribution to the effective action by performing this path integral. Higher terms can then be obtained by contracting the world-line fields with the appropriate Green's function.

Writing for brevity $\tilde{V}(x)=V^{\prime \prime}(\varphi(x))$ and expanding around the common starting and end point $q_{\mu}$ of the closed paths $x_{\mu}(\tau)$ we have

$$
\begin{aligned}
\tilde{V}(x(\tau)) & =\left.e^{(x(\tau)-q)_{\mu} \partial_{\mu}} \tilde{V}(x)\right|_{q} \\
& =\tilde{V}(q)+\beta_{\mu}(x(\tau)-q)_{\mu}+\frac{1}{4} \gamma_{\mu \nu}^{2}(x(\tau)-q)_{\mu}(x(\tau)-q)_{\nu}+\tilde{V}_{3}(x(\tau)-q)
\end{aligned}
$$

where we have introduced the abbreviations

$$
\begin{aligned}
\beta_{\mu} & =\left.\partial_{\mu} \tilde{V}(x)\right|_{q} \\
\gamma_{\mu \nu}^{2} & =\left(\gamma^{2}\right)_{\mu \nu}=\left.2 \partial_{\mu} \partial_{\nu} \tilde{V}(x)\right|_{q} \\
\tilde{V}_{3}(x(\tau)-q) & =\left.\sum_{n=3}^{\infty} \frac{1}{n !}((x(\tau)-q) \cdot \partial)^{n} \tilde{V}(x)\right|_{q}
\end{aligned}
$$


in accordance with ref. 2. Inserting the expansion (2.2) into eq. (2.1), shifting the integration path according to $y_{\mu}(\tau)=x_{\mu}(\tau)-q_{\mu}$ and performing a partial integration in the exponent yields

$$
\begin{aligned}
\Gamma^{(1)}[\varphi] & =\frac{1}{2} \int_{0}^{\infty} \frac{d T}{T} e^{-m^{2} T} \int d^{D} q e^{-\tilde{V}(q) T} \\
& \times \int_{y(0)=0}^{y(T)=0} \mathcal{D} y \exp \left\{-\int_{0}^{T} d \tau\left[-\frac{1}{2} y_{\mu}(\tau)\left(\frac{1}{2} \delta_{\mu \nu} \partial_{\tau}^{2}-\frac{1}{2} \gamma_{\mu \nu}^{2}\right) y_{\nu}(\tau)+\beta_{\mu} y_{\mu}(\tau)+\tilde{V}_{3}(y(\tau))\right]\right\}
\end{aligned}
$$

To further evaluate this world-line path integral we want to get rid of the linear term in the exponent. This is achieved by completing the square with another shift in the integration path

$$
\tilde{y}_{\mu}(\tau)=y_{\mu}(\tau)-\eta_{\mu}(\tau)
$$

where

$$
\eta_{\mu}(\tau)=\int_{0}^{T} d \tau^{\prime} G_{\mu \nu}^{(00)}\left(\tau, \tau^{\prime}\right) \beta_{\nu}=\beta_{\nu} \int_{0}^{T} d \tau^{\prime} G_{\mu \nu}^{(00)}\left(\tau, \tau^{\prime}\right)
$$

and $G^{(00)}$ is the appropriate Green's function defined by the following three properties

$$
\begin{aligned}
\left(\frac{1}{2} \delta_{\mu \nu} \partial_{\tau}^{2}-\frac{1}{2} \gamma_{\mu \nu}^{2}\right) G_{\nu \rho}^{(00)}\left(\tau, \tau^{\prime}\right) & =\delta_{\mu \rho} \delta\left(\tau-\tau^{\prime}\right) \\
G_{\mu \nu}^{(00)}\left(0, \tau^{\prime}\right) & =0 \\
G_{\mu \nu}^{(00)}\left(T, \tau^{\prime}\right) & =0
\end{aligned}
$$

With $G^{(00)}$ satisfying eqs. (2.9) to (2.11) it is ensured that the shifted paths also run from $\tilde{y}(0)=0$ to $\tilde{y}(T)=0$ and that boundary terms vanish. The actual form of $G^{(00)}\left(\tau, \tau^{\prime}\right)$ will be calculated in a moment. For now we can just rely on properties (2.9) to (2.11) to perform the shift. This leads to

$$
\begin{aligned}
\Gamma^{(1)}[\varphi]= & \frac{1}{2} \int_{0}^{\infty} \frac{d T}{T} e^{-m^{2} T} \int d^{D} q e^{-\tilde{V}(q) T} \exp \left\{-\frac{1}{2} \beta_{\mu} \beta_{\nu} \int_{0}^{T} d \tau d \tau^{\prime} G_{\mu \nu}^{(00)}\left(\tau, \tau^{\prime}\right)\right\} \\
& \times \int_{\tilde{y}(0)=0}^{\tilde{y}(T)=0} \mathcal{D} \tilde{y} \exp \left\{-\int_{0}^{T} d \tau\left[-\frac{1}{2} \tilde{y}_{\mu}(\tau)\left(\frac{1}{2} \delta_{\mu \nu} \partial_{\tau}^{2}-\frac{1}{2} \gamma_{\mu \nu}^{2}\right) \tilde{y}_{\nu}(\tau)+\tilde{V}_{3}(\tilde{y}+\eta)\right]\right\}
\end{aligned}
$$

Ignoring the terms of third and higher order in derivatives of the potential, i.e. ignoring $\tilde{V}_{3}$, yields the resummed effective action we are looking for. The influence of these higher derivatives can be taken into account by treating $\tilde{V}_{3}$ as an "interaction term" and calculating the resulting contractions by means of the resummed Green's function $G^{(00)}$.

What remains to complete the calculation is just to perform the quadratic path integral in eq. (2.12) and to determine the Green's function $G^{(00)}$. By the defining property (2.9) the Green's function inverts the operator

$$
K=\frac{1}{2}\left(\mathbb{1} \partial_{\tau}^{2}-\gamma^{2}\right)
$$

which has to be read on the subspace of functions with $x(0)=x(T)=0$ and $x \in \mathbb{R}^{D}$. We now introduce a complete set of one-dimensional normalized eigenfunctions of the operator $\partial_{\tau}^{2}$ that obey the boundary condition $f(0)=f(T)=0$

$$
\left\langle\tau \mid f_{n}\right\rangle=f_{n}(\tau)=\frac{-i}{\sqrt{2 T}}\left(e^{+i \pi n \tau / T}-e^{-i \pi n \tau / T}\right) \quad ; n \in \mathbb{N} \backslash\{0\}
$$


In addition we introduce a set of normalized eigenvectors $\left|v_{1}\right\rangle, \ldots,\left|v_{D}\right\rangle$ of the matrix $\gamma^{2}$. The corresponding eigenvalues $\partial_{\tau}^{2} f_{n}(\tau)=c_{n} f_{n}(\tau)$ are given by

$$
c_{n}=-\frac{\pi^{2} n^{2}}{T^{2}} \quad ; n \in \mathbb{N} \backslash\{0\}
$$

and for the case of the matrix $\gamma^{2}$ will be denoted by $a_{j}$, i.e. $\gamma^{2}\left|v_{j}\right\rangle=a_{j}\left|v_{j}\right\rangle$. With these definitions we have

$$
G^{(00)}=\sum_{n, j} \frac{1}{\frac{1}{2}\left(c_{n}-a_{j}\right)}\left|f_{n}\right\rangle\left|v_{j}\right\rangle\left\langle v_{j}\right|\left\langle f_{n}\right|
$$

and thus

$$
G^{(00)}\left(\tau, \tau^{\prime}\right)=\left\langle\tau\left|G^{(00)}\right| \tau^{\prime}\right\rangle=\sum_{j}\left(\sum_{n} \frac{2 f_{n}(\tau) f_{n}^{*}\left(\tau^{\prime}\right)}{c_{n}-a_{j}}\right)\left|v_{j}\right\rangle\left\langle v_{j}\right|=\sum_{n=1}^{\infty} \frac{2 f_{n}(\tau) f_{n}^{*}\left(\tau^{\prime}\right)}{c_{n} \mathbb{1}-\gamma^{2}}
$$

Inserting in the eigenfunctions and eigenvalues, eqs. (2.14) and (2.15), and using the relation

$$
\sum_{n=1}^{\infty} \frac{\cos n x}{n^{2}+\alpha^{2}}=\frac{\pi}{2 \alpha} \frac{\cosh \alpha(\pi-x)}{\sinh \alpha \pi}-\frac{1}{2 \alpha^{2}} \quad ; 0 \leq x \leq 2 \pi
$$

(8], eq. 1.445.2), we finally arrive at

$$
G^{(00)}\left(\tau, \tau^{\prime}\right)=\frac{\cosh \gamma\left(T-\left|\tau+\tau^{\prime}\right|\right)}{\gamma \sinh \gamma T}-\frac{\cosh \gamma\left(T-\left|\tau-\tau^{\prime}\right|\right)}{\gamma \sinh \gamma T}
$$

which is defined for $0 \leq \tau, \tau^{\prime} \leq T$ and indeed satisfies eqs. (2.9) to (2.11) as can be verified by direct inspection.

To calculate the remaining quadratic path integral in eq. (2.12) we play the usual trick of multiplying and dividing by the known ${ }^{1}$ free path integral

$$
\int_{\tilde{y}(0)=0}^{\tilde{y}(T)=0} \mathcal{D} \tilde{y} \exp \left\{-\int_{0}^{T} d \tau\left[-\frac{1}{2} \tilde{y}_{\mu}(\tau)\left(\frac{1}{2} \delta_{\mu \nu} \partial_{\tau}^{2}\right) \tilde{y}_{\nu}(\tau)\right]\right\}=(4 \pi T)^{-D / 2}
$$

and calculating the resulting quotient of two functional determinants. Thus

$$
\begin{aligned}
I\left(\gamma^{2}, T\right) & =\int_{\tilde{y}(0)=0}^{\tilde{y}(T)=0} \mathcal{D} \tilde{y} \exp \left\{-\int_{0}^{T} d \tau\left[-\frac{1}{2} \tilde{y}_{\mu}(\tau)\left(\frac{1}{2} \delta_{\mu \nu} \partial_{\tau}^{2}-\frac{1}{2} \gamma_{\mu \nu}^{2}\right) \tilde{y}_{\nu}(\tau)\right]\right\} \\
& =(4 \pi T)^{-D / 2} \frac{\operatorname{Det}^{-1 / 2}\left(-\mathbb{1} \partial_{\tau}^{2}+\gamma^{2}\right)}{\operatorname{Det}^{-1 / 2}\left(-\mathbb{1} \partial_{\tau}^{2}\right)}
\end{aligned}
$$

With the identity ( 8 , eq. 1.431.2)

$$
\sinh x=x \cdot \prod_{n=1}^{\infty}\left(1+\frac{x^{2}}{\pi^{2} n^{2}}\right)
$$

and the eigenvalues of $\partial_{\tau}^{2}$ given in eq. (2.15) we find

$$
\begin{aligned}
(4 \pi T)^{D / 2} I\left(\gamma^{2}, T\right) & =\left[\prod_{j=1}^{D} \prod_{n=1}^{\infty}\left(1+\frac{a_{j} T^{2}}{\pi^{2} n^{2}}\right)\right]^{-1 / 2}=\left[\prod_{j=1}^{D} \frac{\sinh \sqrt{a_{j} T^{2}}}{\sqrt{a_{j} T^{2}}}\right]^{-1 / 2} \\
& =\exp \left\{-\frac{1}{2} \sum_{j=1}^{D} \ln \frac{\sinh \sqrt{a_{j} T^{2}}}{\sqrt{a_{j} T^{2}}}\right\}=\exp \left\{-\frac{1}{2} \operatorname{tr} \ln \frac{\sinh \gamma T}{\gamma T}\right\}
\end{aligned}
$$

\footnotetext{
${ }^{1}$ this integral is standard in world-line calculations and can e.g. be evaluated by directly integrating a discretized representation of the path integral
} 
The trace in the last expression is an ordinary matrix trace in D-dimensional Euclidean space.

We can now join together our results for the Green's function and the quadratic path integral, eqs. (2.19) and (2.23), to complete expression (2.12). With the integrated Green's function

$$
\int_{0}^{T} d \tau d \tau^{\prime} G^{(00)}\left(\tau, \tau^{\prime}\right)=-2\left(\frac{T}{\gamma^{2}}-\frac{2}{\gamma^{3}} \tanh \frac{\gamma T}{2}\right)
$$

we obtain the one-loop effective action with the contribution of terms up to second order in the derivative expansion resummed

$$
\Gamma_{\text {res. }}^{(1)}[\varphi]=\int d^{D} q \mathcal{L}_{\text {res. }}^{(1)}[\varphi]=\int d^{D} q \int_{0}^{\infty} d T \mathcal{M}_{\text {res. }}^{(1)}[\varphi]
$$

where

$$
\mathcal{M}_{\text {res. }}^{(1)}[\varphi]=\frac{1}{2(4 \pi)^{D / 2}} \frac{e^{-\alpha T}}{T^{1+D / 2}} \exp \left\{\beta_{\mu}\left(\frac{T}{\gamma^{2}}-\frac{2}{\gamma^{3}} \tanh \frac{\gamma T}{2}\right)_{\mu \nu} \beta_{\nu}-\frac{1}{2} \operatorname{tr} \ln \frac{\sinh \gamma T}{\gamma T}\right\}
$$

and

$$
\alpha=m^{2}+\tilde{V}(q) \quad \beta_{\mu}=\left.\partial_{\mu} \tilde{V}(x)\right|_{q} \quad\left(\gamma^{2}\right)_{\mu \nu}=\left.2 \partial_{\mu} \partial_{\nu} \tilde{V}(x)\right|_{q}
$$

with $\tilde{V}(q)=V^{\prime \prime}(\varphi(q))$ as defined in eqs. (2.3) and (2.4). The complete one-loop effective action including the higher derivative contributions can then be written

$$
\Gamma^{(1)}[\varphi]=\int d^{D} q \int_{0}^{\infty} d T \mathcal{M}_{\text {res. }}^{(1)}[\varphi]\left\langle\exp \left\{-\int_{0}^{T} d \tau \tilde{V}_{3}(\tilde{y}(\tau)+\eta(\tau))\right\}\right\rangle
$$

where $\tilde{V}_{3}$ and $\eta$ have been defined in eqs. (2.5) and (2.8) respectively and Wick contractions are to be performed by means of the resummed Green's function (2.19)

$$
\left\langle\tilde{y}_{\mu}(\tau) \tilde{y}_{\nu}\left(\tau^{\prime}\right)\right\rangle=-G_{\mu \nu}^{(00)}\left(\tau, \tau^{\prime}\right)
$$

Since at first glance it is not completely obvious, we would like to comment in the rest of this section on the equivalence of our result for the resummed one-loop effective Lagrangian, eqs. (2.25) and (2.26), to the one obtained in ref. [2].

For this purpose, it is easiest to start with the expression from ref. [2] (labelled $\mathcal{L}_{2}$ therein). Working in Minkowski space-time they found with $\hbar$ set to unity

$$
\mathcal{L}_{2}=\frac{1}{2(4 \pi)^{2}} \int_{0}^{\infty} d s \frac{1}{s^{3}}\left[\frac{e^{-\alpha s} s^{2}}{\sqrt{\operatorname{det} A_{2}}} e^{-\frac{1}{4} B_{2 \mu}\left(A_{2}^{-1}\right)^{\mu}{ }_{\nu} B_{2}^{\nu}+C_{2}}-e^{-m^{2} s}\right]
$$

where

$$
\begin{aligned}
\left(A_{2}\right)_{\nu}^{\mu} & =\left(\gamma^{-1}\right)_{\rho}^{\mu}(\tan \gamma s)_{\nu}^{\rho} \\
B_{2}^{\mu} & =-2 i\left(\gamma^{-2}\right)_{\rho}^{\mu}\left[g_{\sigma}^{\rho}-(\sec \gamma s)_{\sigma}^{\rho}\right] \beta^{\sigma} \\
C_{2} & =-\frac{1}{2} \operatorname{tr} \ln (\cos \gamma s)-\beta_{\mu}\left(\gamma^{-3}\right)_{\rho}^{\mu}(\tan \gamma s-\gamma s)_{\nu}^{\rho} \beta^{\nu}
\end{aligned}
$$

and $\alpha=m^{2}+\tilde{V}(q)$ as above. Any matrices without indices, e.g. showing up as arguments of the trigonometric functions or in the determinant, have to be understood as build up from the mixed components of the corresponding Lorentz tensors, i.e. first index contravariant, second index covariant. 
Now, using $\ln \operatorname{det} A_{2}=\operatorname{tr} \ln A_{2}$ to lift $A_{2}$ into the exponent and noting $s^{2}=e^{\frac{1}{2} \operatorname{tr} \ln (s \mathbb{1})}$ one can nicely combine the determinant with the tr $\ln$ term in $C_{2}$

$$
\frac{s^{2}}{\sqrt{\operatorname{det} A_{2}}} e^{C_{2}}=\exp \left\{-\frac{1}{2} \operatorname{tr} \ln \frac{\sin \gamma s}{\gamma s}\right\} e^{-\beta_{\mu}\left(\gamma^{-3}\right)^{\mu}{ }_{\rho}(\tan \gamma s-\gamma s)^{\rho}{ }_{\nu} \beta^{\nu}}
$$

The remaining exponential can be written

$$
e^{-\frac{1}{4} B_{2 \mu}\left(A_{2}^{-1}\right)_{\nu}^{\mu} B_{2}^{\nu}}=\exp \left\{\beta_{\mu}\left[\gamma^{-3} \frac{(1-\sec \gamma s)^{2}}{\tan \gamma s}\right]_{\nu}^{\mu} \beta^{\nu}\right\}
$$

where the symmetry of $\gamma_{\mu \nu}^{2}$ leading to $f\left(\gamma^{2}\right)^{\mu}{ }_{\nu}=f\left(\gamma^{2}\right)_{\nu}{ }^{\mu}$ was used. Putting everything together and noting

$$
\frac{(1-\sec \gamma s)^{2}}{\tan \gamma s}-\tan \gamma s=-2 \tan \frac{\gamma s}{2}
$$

one arrives at

$$
\mathcal{L}_{2}=\frac{1}{2(4 \pi)^{2}} \int_{0}^{\infty} \frac{d s}{s^{3}} e^{-\alpha s} \exp \left\{\beta_{\mu}\left(\frac{s}{\gamma^{2}}-\frac{2}{\gamma^{3}} \tan \frac{\gamma s}{2}\right)_{\nu}^{\mu} \beta^{\nu}-\frac{1}{2} \operatorname{tr} \ln \frac{\sin \gamma s}{\gamma s}\right\}
$$

where the field independent $e^{-m^{2} s}$ term that was present in eq. (2.29) and acts as an infrared regulator has been suppressed as in the world-line representation (2.1).

For the final transition from Minkowski to Euclidean space it is essential to remember that $\gamma^{2}$ is really the fundamental object in our equations, not $\gamma$. In fact, one can easily convince oneself that all expressions above when expanded in a power series contain only integer powers of $\gamma^{2}$. Writing the fundamental matrix as $\gamma^{2}$ rather than $\gamma$ is just a device to have such nice shorthand notations as $\gamma^{-3} \tan (\gamma s / 2)$ for a complicated power series in $\gamma^{2}$. With $\tan x=\sum_{n=0}^{\infty} \alpha_{n} x^{2 n+1}$ we have indeed

$$
\frac{2}{\gamma^{3}} \tan \frac{\gamma s}{2}=2 \sum_{n=0}^{\infty} \alpha_{n}\left(\gamma^{2}\right)^{n-1}\left(\frac{s}{2}\right)^{2 n+1}
$$

The product of $n-1$ of the $\gamma^{2}$ matrices contains $n-2$ contracted pairs of Lorentz indices, and therefore transition from Minkowski to Euclidean space-time gives $n-2$ minus signs:

$$
2 \sum_{n=0}^{\infty} \alpha_{n}(-1)^{n-2}\left(\gamma^{2}\right)^{n-1}\left(\frac{s}{2}\right)^{2 n+1}=\frac{2}{\gamma^{3}} \sum_{n=0}^{\infty} \alpha_{n}(-1)^{n}\left(\frac{\gamma s}{2}\right)^{2 n+1}=\frac{2}{\gamma^{3}} \tanh \frac{\gamma s}{2}
$$

Hence, the fact that $\gamma^{2}$ is the fundamental object and not $\gamma$ guarantees that we only get a sign change in every second power of $\gamma$ and thus a transition from tan to $\tanh ^{2}{ }^{2}$ In the same way the sin translates to sinh with an additional minus sign compensated by the trace and we have thereby shown the equivalence of our result (2.26) with eq. (2.29).

\section{Taking different paths}

The calculation of the previous section was based on the path integral representation of the oneloop effective action given in eq. (2.1). This representation contains a path integral over all paths running from $x(0)=q$ to $x(T)=q$ followed by an ordinary integral over all possible positions of the common starting and end point $q$. Together, this corresponds to an integration over the full set of closed paths in configuration space.

We will show now that by choosing a different parametrization of this set a very effective way of resumming terms up to quadratic order in the Taylor expansion of the potential opens up.

\footnotetext{
${ }^{2}$ It is amusing that the same effect is usually triggered by an $i$ coming into play by Wick rotating the Schwinger proper time.
} 
To this end, let us define the "center of mass" of a given path $x(\tau)$ as

$$
q=\frac{1}{T} \int_{0}^{T} d \tau x(\tau)
$$

Doing so, we can alternatively cover the total set of all closed paths by first integrating the paths with a fixed "center of mass" $q$ and afterwards integrating all possible positions of this center. Hence, the representation of the one-loop effective action (2.11) may also be written

$$
\Gamma^{(1)}[\varphi]=\frac{1}{2} \int_{0}^{\infty} \frac{d T}{T} e^{-m^{2} T} \int d^{D} q \mathcal{N}_{(\mathrm{CM}=q)} \mathcal{D} x \exp \left\{-\int_{0}^{T} d \tau\left[\frac{1}{4} \dot{x}^{2}(\tau)+V^{\prime \prime}(\varphi(x))\right]\right\}
$$

The factor $\mathcal{N}^{\prime}$ takes care of a change in the measure accompanying the transition from the path integral with fixed end points to the path integral with fixed "center of mass" (see appendix \$ for details). However, since $\mathcal{N}^{\prime}$ will ultimately drop out of the calculation there is no need to specify it any further here.

Inserting again the expansion (2.2) of the potential, performing a shift in the integration path $y(\tau)=x(\tau)-q$ where now $q$ is the "center of mass" and finally integrating partially in the exponent neglecting the boundary terms leads to

$$
\begin{aligned}
\Gamma^{(1)}[\varphi] & =\frac{1}{2} \int_{0}^{\infty} \frac{d T}{T} e^{-m^{2} T} \int d^{D} q e^{-\tilde{V}(q) T} \\
& \times \mathcal{N}_{(\mathrm{CM}=0)}^{\prime} \mathcal{D} y \exp \left\{-\int_{0}^{T} d \tau\left[-\frac{1}{2} y_{\mu}(\tau)\left(\frac{1}{2} \delta_{\mu \nu} \partial_{\tau}^{2}-\frac{1}{2} \gamma_{\mu \nu}^{2}\right) y_{\nu}(\tau)+\beta_{\mu} y_{\mu}(\tau)+\tilde{V}_{3}(y(\tau))\right]\right\}
\end{aligned}
$$

analogously to eq. (2.6). However, because this time the path integral is performed over the set of closed paths $y(\tau)$ with a vanishing "center of mass", the linear term in the exponent simply disappears being proportional to the integral of $y(\tau)$.

Consequently, following the present approach in resumming terms up to second order we not even have to calculate the Green's function. All that remains is to determine the quadratic path integral

$$
\begin{aligned}
\tilde{I}\left(\gamma^{2}, T\right) & =\mathcal{N}_{(\mathrm{CM}=0)} \oint_{\mathcal{D}} y \exp \left\{-\int_{0}^{T} d \tau\left[-\frac{1}{2} y_{\mu}(\tau)\left(\frac{1}{2} \delta_{\mu \nu} \partial_{\tau}^{2}-\frac{1}{2} \gamma_{\mu \nu}^{2}\right) y_{\nu}(\tau)\right]\right\} \\
& =\mathcal{N}_{(\mathrm{CM}=0)} \oint_{0} \mathcal{D} \exp \left\{-\int_{0}^{T} d \tau \frac{1}{4} \dot{y}^{2}\right\} \frac{\operatorname{Det}_{\mathrm{CM}=0}^{-1 / 2}\left(-\mathbb{1} \partial_{\tau}^{2}+\gamma^{2}\right)}{\operatorname{Det}_{\mathrm{CM}=0}^{-1 / 2}\left(-\mathbb{1} \partial_{\tau}^{2}\right)}
\end{aligned}
$$

At this point the factor $\mathcal{N}^{\prime}$ is reabsorbed by the free path integral transforming back to the free integral with fixed end points which is known to evaluate to $(4 \pi T)^{-D / 2}$ (see appendix @).

The functional determinants are defined on the subspace of closed paths with vanishing "center of mass". A complete set of real valued and normalized one-dimensional eigenfunctions of $\partial_{\tau}^{2}$ with "center of mass" zero and with equal values at $\tau=0$ and $\tau=T$ is given by

$$
\left.\begin{array}{l}
\left\langle\tau \mid \tilde{f}_{n}\right\rangle=\tilde{f}_{n}(\tau)=\frac{-i}{\sqrt{2 T}}\left(e^{+2 \pi i n \tau / T}-e^{-2 \pi i n \tau / T}\right) \\
\left\langle\tau \mid \tilde{g}_{n}\right\rangle=\tilde{g}_{n}(\tau)=\frac{1}{\sqrt{2 T}}\left(e^{+2 \pi i n \tau / T}+e^{-2 \pi i n \tau / T}\right)
\end{array}\right\} \quad ; n \in \mathbb{N} \backslash\{0\}
$$

Hence, there are two linear independent eigenfunctions to any eigenvalue, i.e. $\partial_{\tau}^{2} \tilde{f}_{n}(\tau)=\tilde{c}_{n} \tilde{f}_{n}(\tau)$ and $\partial_{\tau}^{2} \tilde{g}_{n}(\tau)=\tilde{c}_{n} \tilde{g}_{n}(\tau)$ with

$$
\tilde{c}_{n}=-\frac{4 \pi^{2} n^{2}}{T^{2}} \quad ; n \in \mathbb{N} \backslash\{0\}
$$


Using in addition the normalized eigenvectors $\left|v_{1}\right\rangle, \ldots,\left|v_{D}\right\rangle$ of $\gamma^{2}$ with $\gamma^{2}\left|v_{j}\right\rangle=a_{j}\left|v_{j}\right\rangle$ that we already introduced in section 2 the quotient of the two determinants occurring in eq. (3.4) can be evaluated. One finds

$$
\begin{aligned}
(4 \pi T)^{D / 2} \tilde{I}\left(\gamma^{2}, T\right) & =\left[\prod_{j=1}^{D} \prod_{n=1}^{\infty}\left(1+\frac{a_{j} T^{2}}{4 \pi^{2} n^{2}}\right)^{2}\right]^{-1 / 2}=\left[\prod_{j=1}^{D} \frac{\sinh \left(\sqrt{a_{j} T^{2}} / 2\right)}{\sqrt{a_{j} T^{2}} / 2}\right]^{-1} \\
& =\exp \left\{-\sum_{j=1}^{D} \ln \frac{\sinh \left(\sqrt{a_{j} T^{2}} / 2\right)}{\sqrt{a_{j} T^{2}} / 2}\right\}=\exp \left\{-\operatorname{tr} \ln \frac{\sinh (\gamma T / 2)}{\gamma T / 2}\right\}
\end{aligned}
$$

where we again have taken advantage of the identity (2.22).

Thus, for the one-loop effective action with terms up to second order in the derivatives resummed we obtain the alternative expression

$$
\tilde{\Gamma}_{\text {res. }}^{(1)}[\varphi]=\int d^{D} q \tilde{\mathcal{L}}_{\text {res. }}^{(1)}[\varphi]=\int d^{D} q \int_{0}^{\infty} d T \tilde{\mathcal{M}}_{\text {res. }}^{(1)}[\varphi]
$$

with

$$
\tilde{\mathcal{M}}_{\text {res. }}^{(1)}[\varphi]=\frac{1}{2(4 \pi)^{D / 2}} \frac{e^{-\alpha T}}{T^{1+D / 2}} \exp \left\{-\operatorname{tr} \ln \frac{\sinh (\gamma T / 2)}{\gamma T / 2}\right\}
$$

and the same abbreviations as used in eq. (2.26). Accordingly, the complete one-loop effective action may alternatively be written

$$
\Gamma^{(1)}[\varphi]=\int d^{D} q \int_{0}^{\infty} d T \tilde{\mathcal{M}}_{\text {res. }}^{(1)}[\varphi]\left\langle\exp \left\{-\int_{0}^{T} d \tau \tilde{V}_{3}(y(\tau))\right\}\right\rangle
$$

where now contractions of the world-line fields are to be performed with the appropriate Green's function

$$
\left\langle y_{\mu}(\tau) y_{\nu}\left(\tau^{\prime}\right)\right\rangle=-G_{\mu \nu}^{(\mathrm{CM})}\left(\tau, \tau^{\prime}\right)
$$

inverting still the operator $K=\frac{1}{2}\left(\mathbb{1} \partial_{\tau}^{2}-\gamma^{2}\right)$ but this time on the space of closed paths with "center of mass" zero. The construction of $G^{(\mathrm{CM})}$ can be carried out along the same lines as $G^{(00)}$ was obtained in the previous section.

In terms of the eigenstates $\left|\tilde{f}_{n}\right\rangle$ and $\left|\tilde{g}_{n}\right\rangle$ of the operator $\partial_{\tau}^{2}$ in the subspace of functions with "center of mass" zero, eq. (3.5), and the eigenstates $\left|v_{j}\right\rangle$ of the matrix $\gamma^{2}$ one has

$$
G^{(\mathrm{CM})}=\sum_{j=1}^{D} \sum_{n=1}^{\infty} \frac{1}{\frac{1}{2}\left(\tilde{c}_{n}-a_{j}\right)}\left[\left|\tilde{f}_{n}\right\rangle\left|v_{j}\right\rangle\left\langle v_{j}\left|\left\langle\tilde{f}_{n}|+| \tilde{g}_{n}\right\rangle\right| v_{j}\right\rangle\left\langle v_{j}\right|\left\langle\tilde{g}_{n}\right|\right]
$$

and thus

$$
G^{(\mathrm{CM})}\left(\tau, \tau^{\prime}\right)=\left\langle\tau\left|G^{(\mathrm{CM})}\right| \tau^{\prime}\right\rangle=\sum_{n=1}^{\infty} \frac{2\left[\tilde{f}_{n}(\tau) \tilde{f}_{n}^{*}\left(\tau^{\prime}\right)+\tilde{g}_{n}(\tau) \tilde{g}_{n}^{*}\left(\tau^{\prime}\right)\right]}{\tilde{c}_{n} \mathbb{1}-\gamma^{2}}
$$

Finally, inserting the functions $\tilde{f}_{n}(\tau)$ and $\tilde{g}_{n}(\tau)$ from eq. 3.5 leading to

$$
\tilde{f}_{n}(\tau) \tilde{f}_{n}^{*}\left(\tau^{\prime}\right)+\tilde{g}_{n}(\tau) \tilde{g}_{n}^{*}\left(\tau^{\prime}\right)=\frac{2}{T} \cos \left(2 \pi n \frac{\tau-\tau^{\prime}}{T}\right)
$$

and using for a second time the relation (2.18) and the eigenvalues $\tilde{c}_{n}$ from eq. (3.6) yields

$$
G^{(\mathrm{CM})}\left(\tau, \tau^{\prime}\right)=-\frac{\cosh \gamma\left(T / 2-\left|\tau-\tau^{\prime}\right|\right)}{\gamma \sinh (\gamma T / 2)}+\frac{2}{\gamma^{2} T}
$$

Note that

$$
\frac{1}{2}\left(\mathbb{1} \partial_{\tau}^{2}-\gamma^{2}\right) G^{(\mathrm{CM})}\left(\tau, \tau^{\prime}\right)=\mathbb{1}\left(\delta\left(\tau-\tau^{\prime}\right)-\frac{1}{T}\right)
$$


Hence, $G^{(\mathrm{CM})}\left(\tau, \tau^{\prime}\right)$ as given in eq. (3.15) indeed only inverts the operator $K$ in the subspace of paths with a vanishing "center of mass". However, this is all we need. In combination with $G_{\mu \nu}^{(\mathrm{CM})}\left(\tau, \tau^{\prime}\right)=G_{\nu \mu}^{(\mathrm{CM})}\left(\tau^{\prime}, \tau\right), G_{\mu \nu}^{(\mathrm{CM})}\left(0, \tau^{\prime}\right)=G_{\mu \nu}^{(\mathrm{CM})}\left(T, \tau^{\prime}\right), \dot{G}_{\mu \nu}^{(\mathrm{CM})}\left(0, \tau^{\prime}\right)=\dot{G}_{\mu \nu}^{(\mathrm{CM})}\left(T, \tau^{\prime}\right)$ (dots indicating derivatives with respect to the first argument) and $\int_{0}^{T} d \tau G_{\mu \nu}^{(\mathrm{CM})}\left(\tau, \tau^{\prime}\right)=0$, it has all the properties necessary to justify eq. (3.11).

\section{Comparison with the "inverse mass expansion"}

In the previous two sections we derived two expressions for the one-loop effective action, eqs. (2.27) and (3.10). Each of them consists of a resummed part containing the total contribution of a certain subclass of terms and a remainder of individual terms still to be summed up by hand in the form of Wick contractions of the world-line fields.

In order to clarify the relation between the two resummed effective actions $\Gamma_{\text {res. }}^{(1)}$ and $\tilde{\Gamma}_{\text {res. }}^{(1)}$, eqs. (2.25), (2.26) and (3.8), (3.9) respectively, and to have another crosscheck of the calculation we now compare our results to the "inverse mass expansion" discussed in refs. [6, 7].

The "inverse mass expansion" is an expansion of the effective action in powers of the parameter $T$ which has mass dimension -2 . For the case of a real scalar field that we are considering here, the general results in refs. [6] [] reduce to

$$
\Gamma^{(1)}[\varphi]=\frac{1}{2} \int_{0}^{\infty} \frac{d T}{T} \frac{e^{-m^{2} T}}{(4 \pi T)^{D / 2}} \sum_{n=0}^{\infty} \frac{(-T)^{n}}{n !} \int d^{D} q \hat{O}_{n}
$$

where the $\hat{O}_{n}$ up to $n=5$ are given by

$$
\begin{aligned}
& \hat{O}_{0}=1 \\
& \hat{O}_{1}=\tilde{V} \\
& \hat{O}_{2}=\tilde{V}^{2} \\
& \hat{O}_{3}=\tilde{V}^{3}+\frac{1}{2} \tilde{V}_{\mu} \tilde{V}_{\mu} \\
& \hat{O}_{4}=\tilde{V}^{4}+2 \tilde{V} \tilde{V}_{\mu} \tilde{V}_{\mu}+\frac{1}{5} \tilde{V}_{\mu \nu} \tilde{V}_{\mu \nu} \\
& \hat{O}_{5}=\tilde{V}^{5}+5 \tilde{V}^{2} \tilde{V}_{\mu} \tilde{V}_{\mu}+\tilde{V} \tilde{V}_{\mu \nu} \tilde{V}_{\mu \nu}+\frac{5}{3} \tilde{V}_{\mu} \tilde{V}_{\nu} \tilde{V}_{\mu \nu}+\frac{1}{14} \tilde{V}_{\kappa \mu \nu} \tilde{V}_{\kappa \mu \nu}
\end{aligned}
$$

and the shorthand notation $\tilde{V}_{\mu}=\partial_{\mu} \tilde{V}, \tilde{V}_{\mu \nu}=\partial_{\mu} \partial_{\nu} \tilde{V}$ etc. is used with $\tilde{V}$ and its derivatives evaluated at the point $q$.

In comparing $\Gamma_{\text {res. }}^{(1)}$ and $\tilde{\Gamma}_{\text {res. }}^{(1)}$ with the "invers mass expansion" one has to take into account that (4.1) is an expansion of the full one-loop effective action, whereas the resummed effective actions $\Gamma_{\text {res. and }}^{(1)} \tilde{\Gamma}_{\text {res. }}^{(1)}$ only contain certain subclasses of terms. Hence, an expansion of $\Gamma_{\text {res. or }}^{(1)}$ $\tilde{\Gamma}_{\text {res. }}^{(1)}$ in powers of $T$ will in general not lead to the coefficients (4.2) belonging to the full one-loop effective action.

However, if we confine ourselves to the case where third and higher derivatives of $\tilde{V}$ vanish, then the term $\tilde{V}_{3}$ in eqs. (2.27) and (3.10) is zero and both resummed expressions $\Gamma_{\text {res. and }}^{(1)} \tilde{\Gamma}_{\text {res. }}^{(1)}$ coincide with the full one-loop effective action in this case. Consequently, the expansions of $\Gamma_{\text {res. }}^{(1)}$ and $\tilde{\Gamma}_{\text {res. }}^{(1)}$ in powers of $T$ should lead to coefficients, at least consistent with (4.2) under the assumption of vanishing third and higher derivatives of $\tilde{V}$.

Let us check, if this is true. Expanding $\Gamma_{\text {res. }}^{(1)}$ analogously to the expansion of $\Gamma^{(1)}$ in eq. (4.1) 
yields

$$
\begin{aligned}
& O_{0}=1 \\
& O_{1}=\tilde{V} \\
& O_{2}=\tilde{V}^{2}-\frac{1}{3} \tilde{V}_{\mu \mu} \\
& O_{3}=\tilde{V}^{3}-\tilde{V} \tilde{V}_{\mu \mu}-\frac{1}{2} \tilde{V}_{\mu} \tilde{V}_{\mu} \\
& O_{4}=\tilde{V}^{4}-2 \tilde{V}^{2} \tilde{V}_{\mu \mu}-2 \tilde{V} \tilde{V}_{\mu} \tilde{V}_{\mu}+\frac{1}{3} \tilde{V}_{\mu \mu} \tilde{V}_{\nu \nu}+\frac{4}{15} \tilde{V}_{\mu \nu} \tilde{V}_{\mu \nu} \\
& O_{5}=\tilde{V}^{5}-\frac{10}{3} \tilde{V}^{3} \tilde{V}_{\mu \mu}-5 \tilde{V}^{2} \tilde{V}_{\mu} \tilde{V}_{\mu}+\frac{5}{3} \tilde{V} \tilde{V}_{\mu \mu} \tilde{V}_{\nu \nu}+\frac{4}{3} \tilde{V} \tilde{V}_{\mu \nu} \tilde{V}_{\mu \nu}+\frac{5}{3} \tilde{V}_{\mu} \tilde{V}_{\mu} \tilde{V}_{\nu \nu}+2 \tilde{V}_{\mu} \tilde{V}_{\nu} \tilde{V}_{\mu \nu}
\end{aligned}
$$

Accordingly, $\tilde{\Gamma}_{\text {res. }}^{(1)}$ leads to the coefficients

$$
\begin{aligned}
& \tilde{O}_{0}=1 \\
& \tilde{O}_{1}=\tilde{V} \\
& \tilde{O}_{2}=\tilde{V}^{2}-\frac{1}{6} \tilde{V}_{\mu \mu} \\
& \tilde{O}_{3}=\tilde{V}^{3}-\frac{1}{2} \tilde{V} \tilde{V}_{\mu \mu} \\
& \tilde{O}_{4}=\tilde{V}^{4}-\tilde{V}^{2} \tilde{V}_{\mu \mu}+\frac{1}{12} \tilde{V}_{\mu \mu} \tilde{V}_{\nu \nu}+\frac{1}{30} \tilde{V}_{\mu \nu} \tilde{V}_{\mu \nu} \\
& \tilde{O}_{5}=\tilde{V}^{5}-\frac{5}{3} \tilde{V}^{3} \tilde{V}_{\mu \mu}+\frac{5}{12} \tilde{V} \tilde{V}_{\mu \mu} \tilde{V}_{\nu \nu}+\frac{1}{6} \tilde{V} \tilde{V}_{\mu \nu} \tilde{V}_{\mu \nu}
\end{aligned}
$$

To compare eqs. (4.2) - (4.4) one has to recall that these coefficients appear under an integral over $q$ in eq. (4.1) which means that apparently different looking terms can be transformed into each other by partial integrations.

The $\tilde{V}_{\mu \mu}$ term in $O_{2}$ and $\tilde{O}_{2}$, for instance, is a boundary term with respect to this integration.

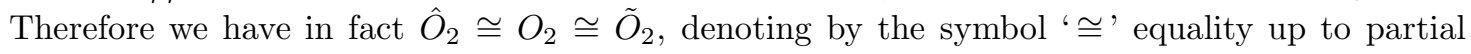
integrations. In the same way we find

$$
-\tilde{V} \tilde{V}_{\mu \mu}-\frac{1}{2} \tilde{V}_{\mu} \tilde{V}_{\mu} \cong-\tilde{V} \tilde{V}_{\mu \mu}+\frac{1}{2} \tilde{V} \tilde{V}_{\mu \mu}=-\frac{1}{2} \tilde{V} \tilde{V}_{\mu \mu} \cong \quad+\frac{1}{2} \tilde{V}_{\mu} \tilde{V}_{\mu}
$$

i.e. $O_{3} \cong \tilde{O}_{3} \cong \hat{O}_{3}$. Inspecting the fourth order terms we can use $\tilde{V}^{2} \tilde{V}_{\mu \mu} \cong-2 \tilde{V}_{\mu} \tilde{V}_{\mu}$ and the fact that under the assumption of vanishing third derivatives $\tilde{V}_{\mu \nu} \tilde{V}_{\mu \nu} \cong 0$ as well as $\tilde{V}_{\mu \mu} \tilde{V}_{\nu \nu} \cong 0$ to establish $O_{4} \cong \tilde{O}_{4} \cong \hat{O}_{4}$. Finally, using $\tilde{V}^{3} \tilde{V}_{\mu \mu} \cong-3 \tilde{V}^{2} V_{\mu} V_{\mu}$ and the three replacements (valid for third derivatives set to zero) $\tilde{V} \tilde{V}_{\mu \nu} \tilde{V}_{\mu \nu} \cong-V_{\mu} V_{\nu} \tilde{V}_{\mu \nu}, \tilde{V} \tilde{V}_{\mu \mu} \tilde{V}_{\nu \nu} \cong+2 V_{\mu} V_{\nu} \tilde{V}_{\mu \nu}$ and $\tilde{V}_{\mu} \tilde{V}_{\mu} \tilde{V}_{\nu \nu} \cong-2 V_{\mu} V_{\nu} \tilde{V}_{\mu \nu}$, one finds $O_{5} \cong \tilde{O}_{5} \cong \hat{O}_{5}$.

Thus, we have shown that under the assumption of vanishing third and higher derivatives the first non-trivial terms of the expansions of $\Gamma_{\text {res. }}^{(1)}$ and $\tilde{\Gamma}_{\text {res. }}^{(1)}$ in powers of $T$ do indeed coincide with the "inverse mass expansion" (4.1), (4.2) of the full one-loop effective action. This is in agreement with the relation $\Gamma^{(1)}=\Gamma_{\text {res. }}^{(1)}=\tilde{\Gamma}_{\text {res. }}^{(1)}$ being a simple consequence of eqs. (2.27) and (3.10) in this case and it serves as a crosscheck that we did not make any calculational errors in deriving the explicit expressions for the resummed effective actions, eqs. (2.25), (2.26) and (3.8), (3.9).

However, there is something else we can learn from the expansions (4.3) and (4.4). The assumption of vanishing third and higher derivatives was imposed above to open up an easy way of comparing the resummed actions $\Gamma_{\text {res. }}^{(1)}$ and $\tilde{\Gamma}_{\text {res. }}^{(1)}$ to the well-known "inverse mass expansion" of the full one-loop effective action. Without this assumption we would have been forced to take into account the contributions from the contractions of world-line fields in eqs. (2.27) and (3.10). However, in comparing $\Gamma_{\text {res. }}^{(1)}$ and $\tilde{\Gamma}_{\text {res. }}^{(1)}$ with each other, there is no need to confine oneself to this special case.

Therefore, let us compare the coefficients (4.3) and (4.4) without assuming third derivatives being zero. We have only referred to this assumption in the discussion of the fourth and fifth order terms, i.e. we still have $O_{n}=\tilde{O}_{n}$ for $n=0,1,2,3$. Using $\tilde{V}_{\mu \mu} \tilde{V}_{\nu \nu} \cong-\tilde{V}_{\mu} \tilde{V}_{\mu \nu \nu} \cong \tilde{V}_{\mu \nu} \tilde{V}_{\mu \nu}$ together with the replacements $\tilde{V}_{\mu} \tilde{V}_{\mu} \tilde{V}_{\nu \nu} \cong-2 \tilde{V}_{\mu} \tilde{V}_{\nu} \tilde{V}_{\mu \nu}$ and $\tilde{V} \tilde{V}_{\mu \nu} \tilde{V}_{\mu \nu} \cong-\tilde{V}_{\mu} \tilde{V}_{\nu} \tilde{V}_{\mu \nu}-\tilde{V} \tilde{V}_{\nu} \tilde{V}_{\mu \mu \nu} \cong$ $-\tilde{V}_{\mu} \tilde{V}_{\nu} \tilde{V}_{\mu \nu}+\tilde{V}_{\nu} \tilde{V}_{\nu} \tilde{V}_{\mu \mu}+\tilde{V}_{\nu \nu} \tilde{V}_{\mu \mu} \cong-3 \tilde{V}_{\mu} \tilde{V}_{\nu} \tilde{V}_{\mu \nu}+\tilde{V} \tilde{V}_{\mu \mu} \tilde{V}_{\nu \nu}$ leads to the following two expressions 
for the fourth order terms

$$
\begin{aligned}
& O_{4} \cong \tilde{V}^{4}+2 \tilde{V} \tilde{V}_{\mu} \tilde{V}_{\mu}+\frac{3}{5} \tilde{V}_{\mu \mu} \tilde{V}_{\nu \nu} \\
& \tilde{O}_{4} \cong \tilde{V}^{4}+2 \tilde{V} \tilde{V}_{\mu} \tilde{V}_{\mu}+\frac{7}{60} \tilde{V}_{\mu \mu} \tilde{V}_{\nu \nu}
\end{aligned}
$$

and to

$$
\begin{aligned}
& O_{5} \cong \tilde{V}^{5}+5 \tilde{V}^{2} \tilde{V}_{\mu} \tilde{V}_{\mu}+3 \tilde{V} \tilde{V}_{\mu \mu} \tilde{V}_{\nu \nu}-\frac{16}{3} \tilde{V}_{\mu} \tilde{V}_{\nu} \tilde{V}_{\mu \nu} \\
& \tilde{O}_{5} \cong \tilde{V}^{5}+5 \tilde{V}^{2} \tilde{V}_{\mu} \tilde{V}_{\mu}+\frac{7}{12} \tilde{V} \tilde{V}_{\mu \mu} \tilde{V}_{\nu \nu}-\frac{1}{2} \tilde{V}_{\mu} \tilde{V}_{\nu} \tilde{V}_{\mu \nu}
\end{aligned}
$$

at fifth order. Thus, in general, we have $\Gamma_{\text {res. }}^{(1)}[\varphi] \neq \tilde{\Gamma}_{\text {res. }}^{(1)}[\varphi]$ which indicates that by the transition to the world-line integral of paths with "center of mass" zero also the class of terms is changed that is taken into account by the resummation.

One may wonder if this does not contradict our derivation of $\Gamma_{\text {res. }}^{(1)}$ and $\tilde{\Gamma}_{\text {res. }}^{(1)}$ which seems to suggest that not only the two representations of the full one-loop effective action, eqs. (2.12) and (3.3), should be equivalent, but also the two expressions for the resummed parts which follow from the full expressions (2.12) and (3.3) by neglecting $\tilde{V}_{3}$ in each of them.

However, there is a subtle point to observe. Indeed, starting from eq. (2.12) without the $\tilde{V}_{3}$ term and tracing back the steps of the derivation one arives at

$$
\begin{aligned}
\Gamma_{\text {res. }}^{(1)}[\varphi] & =\frac{1}{2} \int_{0}^{\infty} \frac{d T}{T} e^{-m^{2} T} \int d^{D} q \\
& \times \int_{x(0)=q}^{x(T)=q} \mathcal{D} x \exp \left\{-\int_{0}^{T} d \tau\left[\frac{1}{4} \dot{x}^{2}+\tilde{V}(q)+\beta_{\mu}(x-q)_{\mu}+\frac{1}{4} \gamma_{\mu \nu}^{2}(x-q)_{\mu}(x-q)_{\nu}\right]\right\}
\end{aligned}
$$

In the same way, starting with eq. (3.3) and dropping $\tilde{V}_{3}$ leads back to

$$
\begin{aligned}
\tilde{\Gamma}_{\text {res. }}^{(1)}[\varphi] & =\frac{1}{2} \int_{0}^{\infty} \frac{d T}{T} e^{-m^{2} T} \int d^{D} q \\
& \times \mathcal{N}_{(\mathrm{CM}=q)}^{\prime} \mathcal{D} x \exp \left\{-\int_{0}^{T} d \tau\left[\frac{1}{4} \dot{x}^{2}+\tilde{V}(q)+\beta_{\mu}(x-q)_{\mu}+\frac{1}{4} \gamma_{\mu \nu}^{2}(x-q)_{\mu}(x-q)_{\nu}\right]\right\}
\end{aligned}
$$

Hence, changing the integration

$$
\int d^{D} q \int_{x(0)=q}^{x(T)=q} \mathcal{D} x \quad \longrightarrow \quad \mathcal{N}^{\prime} \int d^{D} q \oint_{(\mathrm{CM}=q)} \mathcal{D} x
$$

would imply $\Gamma_{\text {res. }}^{(1)}[\varphi]=\tilde{\Gamma}_{\text {res. }}^{(1)}[\varphi]$ in contradiction to what we found above.

The solution to this puzzle is that the change of integration paths (4.12) is not allowed in the transition from eq. (4.10) to eq. (4.11), because the integrand has an explicit $q$ dependence. This becomes clear from our investigations in appendix $\$$ where we justify this change of integration paths: By assuming an additional explicit $q$ dependence (denoted by the last argument after the semicolon) eq. (A.4) changes to

$$
\int d^{D} q \int_{x(0)=q}^{x(T)=q} \mathcal{D} x F[x]=\mathcal{N} \int d^{D} q \int\left[\prod_{m=1}^{N} d^{D} x_{m}\right] f\left(q, x_{1}, \ldots, x_{N}, q ; q\right)
$$


Then, following the same steps as described in appendix Aone obtains instead of eq. A.7)

$$
\int d^{D} q \int_{x(0)=q}^{x(T)=q} \mathcal{D} x F[x]=\int d^{D} c \mathcal{N}^{\prime} \mathcal{N} \int\left[\prod_{m=0}^{N-1} d^{D} x_{m}\right] f\left(x_{0}, x_{1}, \ldots, x_{N}\left(c, x_{0}, \ldots, x_{N-1}\right), x_{0} ; x_{0}\right)
$$

Thus, though we have performed the transition to integration paths with "center of mass" $c$ the explicit parameter dependence of the integrand is still evaluated at $x_{0}$, not at the center $c$. Therefore eqs. (4.10) and (4.11) are not equivalent in general.

On the other hand, in the case of the full one-loop effective action though we write the potential as a Taylor expansion about the point $q$ there is no actual $q$ dependence, because we keep all terms of this expansion. The same is true under the assumption of vanishing third and higher derivatives: Then the terms up to second order already make up the full Taylor expansion and thus the $q$ dependence is only apparent which explains why $\Gamma_{\text {res. }}^{(1)}$ and $\tilde{\Gamma}_{\text {res. }}^{(1)}$ coincide in this case.

\section{Discussion and conclusion}

We have calculated the one-loop contribution to the scalar effective action with terms up to quadratic order in the Taylor expansion of the potential resummed exactly. Using world-line methods we could reproduce an earlier result of E. Masso and F. Rota 2] in a straight forward way. We obtained a representation of the full one-loop effective action with a simple structure (eq. (2.27) ): the full expression consists of the resummed part containing the total contribution of terms up to second order and of a remainder containing the contribution of higher derivatives. This remainder can easily be taken into account to any order desired by calculating Wick contractions of the world-line fields with the appropriate Green's function.

Using a different parametrization of the world-line paths we deduced an alternative representation of the full one-loop effective action in terms of a resummed contribution and a contribution of Wick contracted world-line fields (eq. (3.10)).

If third and higher derivatives of the potential can be neglected both resummed contributions coincide (and are equal to the full one-loop effective action, of course). However, the expression obtained via the second approach, eq. (3.8), has the advantage of being much simpler.

If third and higher derivatives can not be neglected, the two resummed expressions, eqs. (2.25) and (3.8) are no longer equivalent, though the combinations of each resummed part with the contribution coming from the respective Wick contractions are. Thus, to calculate the oneloop effective action to a given order one can either take the resummed contribution and Wick contractions of the first approach or of the second. Again, the second alternative seems preferable, because the resummed contribution as well as the Green's function is simpler in this case.

Finally, let us mention that the method presented here can equally well be applied to the case of gauge fields. In the abelian case for instance, starting from the world-line representation of the one-loop effective action in Fock-Schwinger gauge (see ref. [7])

$$
\begin{aligned}
\Gamma^{(1)}[A] & =\int_{0}^{\infty} \frac{d T}{T} e^{-m^{2} T} \int d^{D} q \\
& \times \mathcal{N}_{(\mathrm{CM}=0)}^{\prime} \mathcal{D} y \exp \left\{-\int_{0}^{T} d \tau\left[\frac{1}{4} \dot{y}^{2}(\tau)+i e \int_{0}^{1} d \eta \eta y_{\mu}(\tau) \mathcal{F}_{\mu \nu}(q+\eta y(\tau)) \dot{y}_{\nu}(\tau)\right]\right\}
\end{aligned}
$$

and inserting for the field strength tensor its gauge covariant expansion (see, e.g. 9])

$$
\mathcal{F}_{\mu \nu}(q+\eta y(\tau))=e^{\eta y(\tau) \cdot D} \mathcal{F}_{\mu \nu}(q)
$$


one obtains

$$
\begin{aligned}
\Gamma^{(1)}[A] & =\int_{0}^{\infty} \frac{d T}{T} e^{-m^{2} T} \int d^{D} q \\
& \times \mathcal{N}_{(\mathrm{CM}=0)} \mathcal{D} y \exp \left\{-\int_{0}^{T} d \tau\left[-\frac{1}{2} y_{\mu}(\tau)\left(\frac{1}{2} \delta_{\mu \nu} \partial_{\tau}^{2}-i e \mathcal{F}_{\mu \nu}(q) \partial_{\tau}\right) y_{\nu}(\tau)+\tilde{V}_{3}(q, y(\tau))\right]\right\}
\end{aligned}
$$

Here we have extracted the first term of the expansion which in the case of a gauge field is already quadratic in the world-line field $y(\tau)$ and therefore is the contribution that can be resummed exactly. The remaining terms of the expansion are collected in

$$
\tilde{V}_{3}(q, y(\tau))=\sum_{n=1}^{\infty} \frac{i e}{n !(n+2)} y_{\mu}(y \cdot D)^{n} \mathcal{F}_{\mu \nu}(q) \dot{y}_{\nu}
$$

as before. If the field strength is constant, $\tilde{V}_{3}(q, y(\tau))$ vanishs and thus the resummed effective action can immediately be read off from the corresponding Euler-Heisenberg action [10] which is a well-known object within the world-line formalism [11, 12, 13. With the result for the one-loop case in refs. 4, 11] we find

$$
\Gamma_{\text {res. }}^{(1)}[A]=\int d^{D} q \mathcal{L}_{\text {res. }}^{(1)}[A]=\int d^{D} q \int_{0}^{\infty} d T \mathcal{M}_{\text {res. }}^{(1)}[A]
$$

with

$$
\mathcal{M}_{\text {res. }}^{(1)}[A]=\frac{1}{(4 \pi)^{D / 2}} \frac{e^{-m^{2} T}}{T^{1+D / 2}} \exp \left\{-\frac{1}{2} \operatorname{tr} \ln \frac{\sin e \mathcal{F}(q) T}{e \mathcal{F}(q) T}\right\}
$$

If the assumption of a slowly varying field is not justified, the effect of $\tilde{V}_{3}(q, y(\tau))$ has to be taken into account. As we have seen, in the present formalism this can easily be achieved by calculating the corresponding Wick contractions of the world-line fields by means of the resummed Green's function, i.e.

$$
\Gamma^{(1)}[A]=\int d^{D} q \int_{0}^{\infty} d T \mathcal{M}_{\text {res. }}^{(1)}[A]\left\langle\exp \left\{-\int_{0}^{T} d \tau \tilde{V}_{3}(q, y(\tau))\right\}\right\rangle
$$

with

$$
\left\langle y_{\mu}(\tau) y_{\nu}\left(\tau^{\prime}\right)\right\rangle=-\mathcal{G}_{\mu \nu}^{(B)}\left(\tau, \tau^{\prime}\right)
$$

and (see ref. [1])

$$
\mathcal{G}^{(B)}\left(\tau, \tau^{\prime}\right)=\frac{1}{2(e \mathcal{F})^{2}}\left(\frac{e \mathcal{F}}{\sin e \mathcal{F} T} e^{-i e \mathcal{F} T \dot{G}_{B}\left(\tau, \tau^{\prime}\right)}+i e \mathcal{F} \dot{G}_{B}\left(\tau, \tau^{\prime}\right)-\frac{1}{T}\right)
$$

where $\dot{G}_{B}\left(\tau, \tau^{\prime}\right)=\partial_{\tau} G_{B}\left(\tau, \tau^{\prime}\right)$ and $G_{B}\left(\tau, \tau^{\prime}\right)=\left|\tau-\tau^{\prime}\right|-\left(\tau-\tau^{\prime}\right)^{2} / T$. This then is also a very economised reorganization of the invers mass expansion in ref. [7].

\section{A Fixed end points versus fixed center}

In this appendix we make a few comments on the relation between world-line path integrals over the class of all closed paths with a fixed starting and end point $x(0)=q=x(T)$ and path integrals over the class of all closed paths with a fixed "center of mass"

$$
q=\frac{1}{T} \int_{0}^{T} d \tau x(\tau)
$$


Originally, the path integrals encountered in the world-line formalism are of the first kind. The representation of the one-loop effective action, eq. 2.1), for instance is basically the trace of some operator evaluated in configuration space

$$
\operatorname{Tr}\{O\}=\int d^{D} q\langle q|O| q\rangle
$$

The path integral then arises by introducing complete sets of intermediate states between $x(0)=q$ and $x(T)=q$. Thus, one typically obtains a path integral over the set of paths with fixed starting and end point $q$ that is followed by an ordinary integral over all possible positions of the point $q$. In conjunction, this makes up an integration over all closed paths as such, i.e. without any further restriction.

However, as we have seen in section 3 sometimes it proves useful to parametrize this integration over the total set of closed paths in a different way: defining the "center of mass" of a given path via eq. (A.1) one first integrates over the subset of paths with a fixed "center of mass" $q$. Afterwards, one integrates all possible positions of this center. Obviously, this second procedure also comprises an integration over the totality of closed paths.

However, one should be aware that depending on the precise definition of "integral over all paths with center $q$ " in terms of a discretization ${ }^{3}$ there may be a change in the path integral measure accompanying the transition from the path integral with fixed end points to the path integral with fixed center.

To explain this issue let $F[x]$ be a functional defined for paths $x:[0, T] \rightarrow \mathbb{R}^{D}, \tau \mapsto x(\tau)$. We choose a discretization where the paths are described by the starting point $x_{0}=x(0)$, the end point $x_{N+1}=x(T)$ and $N$ intermediate points $x_{1}, \ldots, x_{N}$. Then the functional $F[x]$ is replaced by an ordinary function of the discretization points $f\left(x_{0}, x_{1}, \ldots, x_{N}, x_{N+1}\right)$ and we can define the functional integral as

$$
\int_{x(0)=p}^{x(T)=q} \mathcal{D} x F[x]=\mathcal{N} \int\left[\prod_{m=1}^{N} d^{D} x_{m}\right] f\left(p, x_{1}, \ldots, x_{N}, q\right)
$$

where $\mathcal{N}$ is some given measure. According to this definition we have

$$
\begin{aligned}
\int d^{D} q \int_{x(0)=q}^{x(T)=q} \mathcal{D} x F[x] & =\mathcal{N} \int d^{D} q \int\left[\prod_{m=1}^{N} d^{D} x_{m}\right] f\left(q, x_{1}, \ldots, x_{N}, q\right) \\
& =\mathcal{N} \int\left[\prod_{m=0}^{N} d^{D} x_{m}\right] f\left(x_{0}, x_{1}, \ldots, x_{N}, x_{0}\right)
\end{aligned}
$$

Analogously, the "center of mass" functional translates

$$
C[x]=\frac{1}{T} \int_{0}^{T} d \tau x(\tau) \quad \longrightarrow \quad c\left(x_{0}, \ldots, x_{N}\right)=\frac{1}{N+1} \sum_{k=0}^{N} x_{k}
$$

and can be brought into the game by inserting unity into the integral A.4

$$
\int d^{D} q \int_{x(0)=q}^{x(T)=q} \mathcal{D} x F[x]=\mathcal{N} \int\left[\prod_{m=0}^{N} d^{D} x_{m}\right] \int d^{D} c \delta^{D}\left(c-\frac{1}{N+1} \sum_{k=0}^{N} x_{k}\right) f\left(x_{0}, x_{1}, \ldots, x_{N}, x_{0}\right)
$$

\footnotetext{
${ }^{3}$ After all, any path integral formula should always be understood as a mere shorthand notation for a certain discretization.
} 
Pulling out the factor of $1 /(N+1)$ and using the delta function to perform the integral over one of the intermediate points, say $x_{N}$, one is led to

$$
\int d^{D} q \int_{x(0)=q}^{x(T)=q} \mathcal{D} x F[x]=\int d^{D} c \mathcal{N}^{\prime} \mathcal{N} \int\left[\prod_{m=0}^{N-1} d^{D} x_{m}\right] f\left(x_{0}, x_{1}, \ldots, x_{N}\left(c, x_{0}, \ldots, x_{N-1}\right), x_{0}\right)
$$

with $\mathcal{N}^{\prime}=(N+1)^{D}$ and $x_{N}\left(c, x_{0}, \ldots, x_{N-1}\right)=(N+1) c-\sum_{k=0}^{N-1} x_{k}$. Note that the factor $(N+1)^{D}$ is not a combinatorical factor related to some symmetry of interchanging discretization points. It is simply a consequence of the specific choice of the restriction $c=c\left(x_{0}, \ldots, x_{N}\right)$ that the paths are subject to and would be different for other choices than A.5.

To proceed, let us discuss the expression

$$
\mathcal{N} \int\left[\prod_{m=0}^{N-1} d^{D} x_{m}\right] f\left(x_{0}, x_{1}, \ldots, x_{N}\left(c, x_{0}, \ldots, x_{N-1}\right), x_{0}\right)
$$

occurring on the right-hand side of eq. A.7. It contains $N$ independent integrations over the discretization points $x_{0}, \ldots, x_{N-1}$. Depending on these values, $x_{N}$ is choosen in just the right way to give the whole path a "center of mass" equal to the given value $c$. Finally, $x_{N+1}$ is set to $x_{0}$ constructing a closed path.

Thus, expression A.8 is exactly what one would naturally write down as a discretization of an integral over all closed paths with a fixed "center of mass" equal to $c$. Consequently, eq. A.7. may be written

$$
\int d^{D} q \int_{x(0)=q}^{x(T)=q} \mathcal{D} x F[x]=\mathcal{N}^{\prime} \int d^{D} c \oint_{(\mathrm{CM}=c)} \mathcal{D} x F[x]
$$

and we have shown that the two path integrals are equivalent up to a change in the measure.

However, let us argue now that the factor $\mathcal{N}^{\prime}$ coming into existence in the transition from path integrals with fixed end points to integrals with fixed "center of mass" will in general disappear in the end of the calculation and therefore does not require much attention.

As we have mentioned above a world-line calculation typically starts from a path integral with fixed end points. If it is transformed into an integral with fixed center, a factor $\mathcal{N}^{\prime}$ occurs. However, after some manipulations the path integral will generally end up in an expression proportional to the free integral over closed paths with "center of mass" zero. This free integral finally grabs the factor $\mathcal{N}^{\prime}$ to transform back into a free integral with fixed end points which is the one we know to evaluate to $(4 \pi T)^{-D / 2}$, i.e.

$$
\begin{aligned}
\int d^{D} q \int_{x(0)=q}^{x(T)=q} \mathcal{D} x F[x] & =\mathcal{N}^{\prime} \int^{D} c \oint_{(\mathrm{CM}=c)} \mathcal{D} x F[x] \\
& =\mathcal{N}^{\prime} \oint_{(\mathrm{CM}=0)} \mathcal{D} y \exp \left\{-\int_{0}^{T} d \tau \frac{1}{4} \dot{y}^{2}\right\} \int d^{D} c \text { (some function of } c \text { ) } \\
& =\underbrace{\int_{y(0)=0} \mathcal{D} y \exp \left\{-\int_{0}^{T} d \tau \frac{1}{4} \dot{y}^{2}\right\}}_{(4 \pi T)^{-D / 2}} \int d^{D} c \text { (some function of } c \text { ) }
\end{aligned}
$$

where we have used

$$
\int_{y(0)=0}^{y(T)=0} \mathcal{D} y \exp \left\{-\int_{0}^{T} d \tau \frac{1}{4} \dot{y}^{2}\right\}=\mathcal{N}_{(\mathrm{CM}=0)} \oint_{0} \mathcal{D} y \exp \left\{-\int_{0}^{T} d \tau \frac{1}{4} \dot{y}^{2}\right\}
$$


which is a consequence of eq. (A.9) applied to $F[x]=\exp \left\{-\int_{0}^{T} d \tau \frac{1}{4} \dot{x}^{2}\right\}$ and a shift of the integration paths in each of the two integrals according to $y(\tau)=x(\tau)-q$ and $y(\tau)=x(\tau)-c$ respectively.

\section{References}

[1] M. R. Brown, M. J. Duff, Phys. Rev. D11 (1975) 2124

[2] E. Masso, F. Rota, Nucl. Phys. B620 (2002) 566 arXiv:hep-th/0109046

[3] M. J. Strassler, Nucl. Phys. B385 (1992) 145 arXiv:hep-ph/9205205

[4] M. G. Schmidt, C. Schubert, Phys. Lett. B318 (1993) 438 arXiv:hep-th/9309055.

[5] C. Schubert, Phys. Rep. 355 (2001) 73 arXiv:hep-th/0101036

[6] D. Fliegner, P. Haberl, M. G. Schmidt, C. Schubert, Discourses Math. Appl. 4 (1995) 87 arXiv:hep-th/9411177

[7] D. Fliegner, P. Haberl, M. G. Schmidt, C. Schubert, Annals Phys. 264 (1998) 51 arXiv:hep-th/9707189

[8] I. S. Gradshteyn, I. M. Ryzhik, "Table of Integrals, Series and Products", Academic Press, New York and London, 4th edition, 1965

[9] M. A. Shifman, Nucl. Phys. B173 (1980) 13

[10] W. Heisenberg, H. Euler, Z. Phys. 98 (1936) 714

[11] M. Reuter, M. G. Schmidt, C. Schubert, Annals Phys. 259 (1997) 313 arXiv:hep-th/9610191

[12] B. Körs, M. G. Schmidt, Eur. Phys. J. C6 (1999) 175 arXiv:hep-th/9803144

[13] H.-T. Sato, M. G. Schmidt, C. Zahlten, Nucl. Phys. B579 (2000) 492 arXiv:hep-th/0003070 